
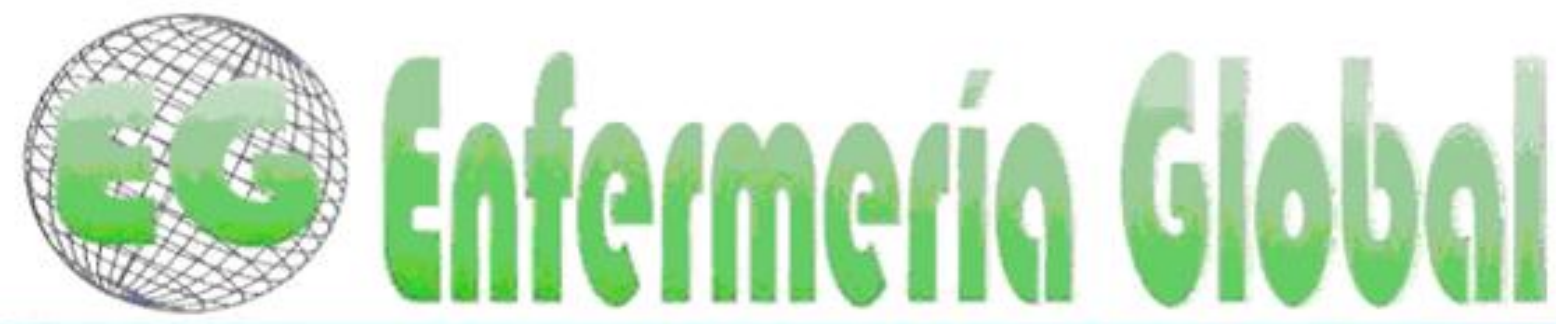

www.um.es/egloball

\title{
CLÍNICA
}

\section{En torno a los hemoderivados}

About blood products

\section{*Cortés Fadrique, Carmen *Del Trigo Méndez, Patricia *Veiga Frá, Rubén *Sánchez Bermejo, Raúl ${ }^{* *}$ Rincón Fraile, Beatriz *Fernández Centeno, Esther}

\author{
*Enfermera/o. Hospital General Nuestra Señora del Prado. Talavera de la Reina. Toledo. E-mail: \\ rsanchezb@sescam.jccm.es **Enfermera. Hospital Virgen de la Salud. Granada. España. \\ Palabras Clave: Hemoderivados; transfusiones; concentrado de hematíes \\ Keywords: Blood products; transfusions; packed red blood cells.
}

\section{RESUMEN}

La transfusión es una necesidad permanente, y la amplitud con la que es utilizada exige que deba garantizarse su calidad y seguridad para evitar, en particular, la transmisión de enfermedades. Ha de ser un tratamiento personalizado. Las funciones de enfermería son de especial importancia así como los cuidados que se requieren.

El objetivo del presente estudio es conocer la variabilidad práctica de los profesionales de enfermería del centro hospitalario, sobre la extracción de muestras pretransfusionales y la administración de hemoderivados.

Para lo cual se realizó el envío de un cuestionario para su posterior cumplimentación en formato online, que garantizaba el total anonimato. Han contestado a la encuesta 180 profesionales. El $74.4 \%$ de los enfermeros dice que la transfusión de hemoderivados sólo se puede administrar de forma simultánea con suero fisiológico. Un $56.1 \%$ refiere que cada concentrado transfundido de hematíes aumenta la hemoglobina en $1 \mathrm{gr} / \mathrm{dl}$. Hemos encontrado un consenso entre las recomendaciones científicas y las contestaciones realizadas por los diferentes profesionales, hecho que se reafirma con el escaso índice de notificaciones adversas que se han registrado en nuestro trabajo.

La elaboración e implantación de una guía de actuación en cuanto a la administración de hemoderivados se hace imprescindible

\section{ABSTRACT}

Transfusion is an ongoing need, and as widely used it requires that quality and safety should be ensured to avoid, in particular, the transmission of diseases. It must be a custom treatment. Nursing roles are particularly important as the care required. The aim of this study is to determine the variability of nursing skills on the extraction of pre-transfusion samples and administration of blood products. 
Anonymous questionnaires were sent out on-line for subsequent filling and 180 nursing professionals participated. $74.4 \%$ of nurses said that blood transfusion can only be administered simultaneously with normal saline, $56.1 \%$ reported that each transfused packed red blood cells increases hemoglobin $1 \mathrm{~g} /$ dl. We found a consensus among the scientific recommendations and the responses made by different professionals, a fact that is confirmed by the low rate of adverse notifications registered in our study. The development and implementation of policy guidance regarding the administration of blood products is essential.

\section{INTRODUCCIÓN}

El volumen de sangre de un adulto normal es, por término medio, del $8 \%$ del peso corporal, es decir, unos 5 litros. Alrededor del $60 \%$ de la sangre está formada por plasma y el $40 \%$ restante lo componen las células sanguíneas ${ }^{1}$.

La transfusión es una necesidad permanente, y la amplitud con la que es utilizada exige que deba garantizarse su calidad y seguridad para evitar, en particular, la transmisión de enfermedades ${ }^{2}$.

La transfusión tiene aspectos legales, éticos, médicos y sociales peculiares, y debe estar basada en el principio de máxima seguridad para el receptor ${ }^{3}$.

El Real Decreto 1088/2005, de 16 de septiembre, por el que se establecen los requisitos técnicos y condiciones mínimas de la hemodonación y de los centros y servicios de transfusión, en el Capítulo IV; artículos 15-20, regula la prescripción y administración de sangre y sus componentes ${ }^{2}$.

La transfusión de productos sanguíneos contribuye diariamente a mejorar la calidad asistencial y, por tanto, a la recuperación total o parcial de la salud de nuestros ciudadanos, pero también, como todos sabemos, puede ser la causa de efectos adversos de gravedad variable ${ }^{4}$.

Ha de ser un tratamiento personalizado. Hay que tener presentes varios factores, como la edad, la enfermedad de base y la sintomatología, entre otros. Se ha de tratar a los pacientes, no a los resultados del laboratorio ${ }^{4}$.

En Europa, el informe SHOT publicado en 2004, sobre un seguimiento de 10 años, muestra una cifra de 4/1.000.000 muertes relacionadas con la transfusión, aunque de ellas, 7/10.000.000 estaban relacionadas con un error en la administración del hemoderivado ${ }^{5}$.

En esencia, se trata de evitar el error de identificación ${ }^{6}$ en cualquiera de las etapas del proceso transfusional (toma de muestra, entrega al banco, dispensación del hemoderivado y administración). El papel de las enfermeras en la mejora de la seguridad transfusional es esencial, al estar presente e involucrada en momentos críticos de este proceso $0^{5,7}$.

La finalidad de la transfusión de hematíes es aumentar la capacidad de transporte de oxígeno a los tejidos gracias a la hemoglobina $(\mathrm{Hb})$. El volumen aproximado del producto se sitúa entre 200 y $300 \mathrm{ml}$. Su indicación es para el tratamiento de la anemia tanto en proceso agudo como crónico ${ }^{4}$. 
Cada unidad de sangre completa $\mathrm{o}$ de concentrado de hematíes contiene $\mathrm{Hb}$ suficiente para elevar, como media, la $\mathrm{Hb}$ del paciente en $1 \mathrm{~g} / \mathrm{dl}$ o en 3 puntos el porcentaje del hematocrito.

En adultos se administrará la dosis mínima, necesaria para eliminar la sintomatología, hemos de huir del concepto clásico: una dosis $=2$ unidades. En muchas ocasiones, un solo concentrado de hematíes es suficiente para aliviar la sintomatología del paciente y proseguir con el tratamiento etiológico. En pacientes de edad avanzada o con problemas cardiovasculares es aconsejable no transfundir más de una unidad de manera sucesiva.

Las plaquetas son elementos sanguíneos esenciales para la detención de las hemorragias. Circulan en número de entre 125 y $300 \times 10^{9} / l^{12}$. Los concentrados de plaquetas se transfunden para prevenir o tratar hemorragias en pacientes con efectos cualitativos y/o cuantitativos de las plaquetas.

Para un adulto, la dosis habitual de concentrados de plaquetas individuales es de un concentrado por cada $10 \mathrm{~kg}$ de peso. Es decir, un adulto requiere entre 5 y 7 unidades.

Los objetivos del estudio son:

a. Objetivo principal:

- Conocer la variabilidad práctica de los DUE's, sobre la extracción de muestras pretransfusionales y la administración de hemoderivados.

b. Objetivos específicos:

- Reconocer las condiciones para la extracción de muestras pretransfusionales y junto con las medidas de seguridad clínica que se adoptan.

- Definir las condiciones de administración de hemoderivados (tiempo, necesidades del paciente...)

\section{MATERIAL Y MÉTODO}

Estudio descriptivo transversal que se realizó en el Hospital General Nuestra Señora del Prado (HGNSP). Consistió en el envío, por correo electrónico, de un "link" que enlaza con una encuesta para autocumplimentación a los profesionales de enfermería que se encuentran trabajando en alguna de las unidades del centro hospitalario, en el momento de la realización del estudio.

El diseño del cuestionario recoge datos que incluyen tanto variables socio demográficas (edad, sexo, unidad de trabajo) como variables para establecer conocimiento acerca de los hemoderivados (finalidad de los hemoderivados, tiempo de validez de las pruebas cruzadas) así como también cuestiones intrínsecas a su realización (identificación, tiempos de transfusión, administración simultánea con otras soluciones, ayunas, temperatura de infusión, presencia de fiebre, actuación ante reacciones transfusionales) (Ver Anexo I) 
Se ha limitado la contestación a la encuesta por equipo, de tal forma que solo se permite la cumplimentación de la encuesta en una única ocasión. El periodo de recepción de las encuestas fue del 1 de Diciembre de 2012 a 1 de Abril de 2013.

Los criterios de inclusión se definieron mediante el envío de la encuesta a todos los profesionales de enfermería que constituían la plantilla del Hospital General Nuestra Señora del Prado, formada por 340 profesionales.

Análisis descriptivo expresado como media, desviación estándar, rango para variables cuantitativas y como valor absoluto y porcentajes para variables cualitativas.

Los datos fueron recogidos en la base de la web e-encuestas.com ( $)$, utilizada para el estudio, donde se asegura el completo anonimato de las personas que participan en el estudio. Posteriormente se realizará el análisis pormenorizado de los datos obtenidos con el programa estadístico SPSS 20.0 ^ y Microsft Office Excel 2007®.

\section{RESULTADOS}

Han contestado a la encuesta 180 diplomados universitarios de enfermería (DUE) de los 340 que forman la plantilla del HGNSP, es decir, un $53 \%$ del total de profesionales de enfermería que componen la plantilla del hospital. De los cuales el $80.6 \%$ han sido mujeres y el $17.2 \%$ hombres. Con una edad media de 37.5 [19-62] años y una experiencia media de $14.5( \pm 8.5)$ años. Estos pertenecían a las unidades de hospitalización (47.8\%) y otros servicios: unidad de apoyo (6.7\%).

Ante el planteamiento de la pregunta: "El tratamiento con la transfusión de hematíes está dirigido, en primer lugar a": el $22.8 \%$ indicó que a garantizar el transporte de oxigeno, el $16.1 \%$ a garantizar la volemia, $1.7 \%$ a la reposición de proteínas plasmáticas y plaquetas y un $42.2 \%$ a garantizar todo lo anterior.

El $60 \%$ de los encuestados indicó que desde el momento de la extracción el tiempo de validez de las pruebas cruzadas el de 48 horas, un $17.8 \%$ no sabía o no contestó, un 12.2 que su validez es de 24 horas y un 6.7 que su validez se prolonga durante la duración del ingreso del paciente.

El $66.7 \%$ de los profesionales conoce cuál es el tubo correcto (tubo Edta: tapón violeta de $9 \mathrm{cc}$ ) que ha de extraer para la realización de las "pruebas cruzadas" previo a la transfusión. El resto indican otros tubos, no saben o no contestan.

Con relación a los tiempos que debe durar la administración de hemoderivados, el 48.3\% indica que 60-120 minutos, nunca más de 6 horas si se trata de concentrados de hematíes y el paciente no presenta ningún patología asociada que lo contraindique y un $20.6 \%$ dice que $120-240$ minutos, nunca más de 4 horas. Si se administran concentrados de plaquetas el $55.6 \%$ refiere que entre $20-30$ minutos y un $13.3 \%$ que entre 45-60 minutos.

En cuanto a si se puede administrar simultáneamente por la misma vía algún tipo de solución endovenosa, el $74.4 \%$ dice que solo suero fisiológico, un $5 \%$ añade que además puede administrarse conjuntamente Voluven ${ }^{\circledR}$, un $1.7 \%$ dice que no puede ser mezclado con ningún otro tipo de solución endovenosa y $18.9 \%$ no sabe o no contesta. 
Un $71.1 \%$ de los profesionales encuestados refiere que para la administración de concentrados de hematíes el paciente no es necesario que permanezca en ayunas.

Referente a la pregunta "¿A qué temperatura se debe transfundir una bolsa de concentrados de hematíes?", el $49.4 \%$ contesta que ha de administrarse según llega del banco de sangre, mientras que un $23.9 \%$ indica que se tiene que esperar a que alcance la temperatura ambiente.

Referente al planteamiento de: "Si antes de iniciar la transfusión, el paciente presenta fiebre. ¿Se puede administrar dicho concentrado de hematíes?". El 36.7\% dice que ha de consultar siempre al Facultativo antes de iniciar la transfusión, un $31.1 \%$ indica que no se puede iniciar dicha transfusión y un $12.2 \%$ refiere que sí se puede iniciar la transfusión. Mientras que si dicha fiebre aparece durante el proceso de transfusión, el $60.6 \%$ indica que la actuación a seguir es parar inmediatamente la transfusión y un $18,9 \%$ que su actuación sería avisar al médico y que indique la actuación a seguir.

En cuanto a "¿Cuál es el volumen habitual que se administra en cada bolsa de concentrado de hematíes?", un $48.9 \%$ dice que entre $200-300 \mathrm{ml}$ por concentrado y el $28.9 \%$ indica que el volumen es de $400-500 \mathrm{ml}$.

Referente a cuáles serían la actuaciones de enfermería a realizar de forma inmediata ante una reacción transfusional, estas quedan recogidas en el gráfico 1

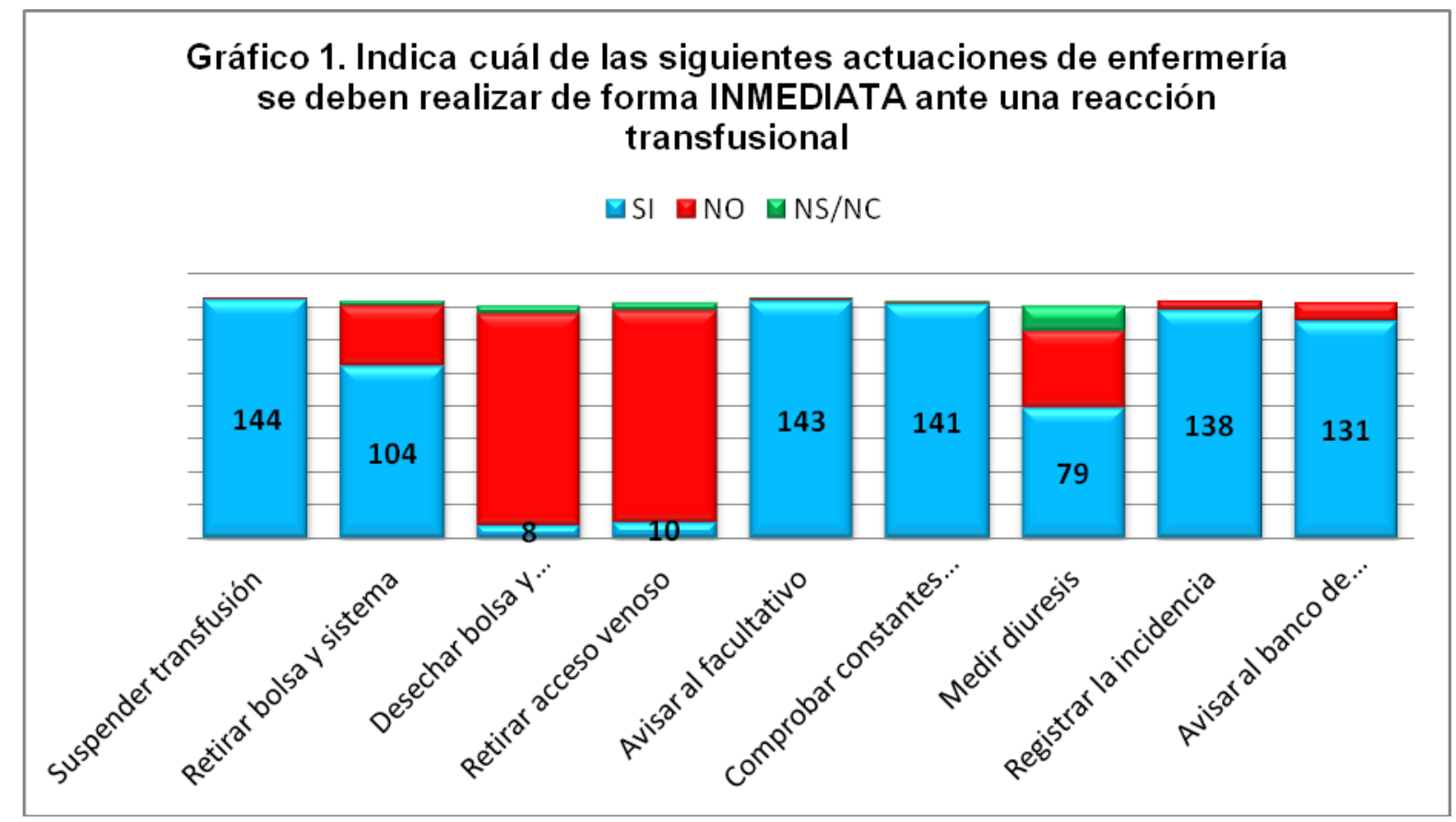

Ante el planteamiento: "Como media, cada concentrado transfundido de hematíes aumenta la hemoglobina:" el $56.1 \%$ refiere que $1 \mathrm{gr} / \mathrm{dl}$, el $16.5 \%$ refiere que $0.5 \mathrm{gr} / \mathrm{dl}$, $15.8 \%$ indica que es necesario poner como mínimo 2 concentrados de hematíes para que la hemoglobina se vea aumentada, un $10.8 \%$ que $3 \mathrm{gr} / \mathrm{dl}$. 


\section{DISCUSIÓN.}

Consideramos un alto índice de respuesta, ya que es superior al $40 \%$ para cuestionarios autocumplimentados. No hemos podido comparar nuestros resultados con estudios de la misma índole, puesto que no hemos encontrado estudios similares en las principales bases de datos.

Hemos encontrado un consenso entre las recomendaciones científicas y las contestaciones realizadas por los diferentes profesionales, hecho que se reafirma con el escaso índice de notificaciones adversas que se han registrado en nuestro trabajo, aunque hemos de decir que por la importancia y relevancia del mismo nos hubiese gustado encontrar unanimidad en cuanto a las contestaciones en algunas cuestiones.

Analizando pormenorizadamente los datos obtenidos observamos algunas cuestiones a destacar:

Ante el planteamiento de la pregunta: "El tratamiento con la transfusión de hematíes está dirigido a", algo menos de un cuarto de los profesionales concluyó que su indicación se encontraba dirigida principalmente a garantizar el transporte de oxigeno, tal y como indica la bibliografía consultada ${ }^{4}$ y no a mantener la volemia ni a la reposición de proteínas plasmáticas y plaquetas.

Podemos deducir que más de la mitad de los profesionales conoce el tiempo de validez de extracción de las pruebas cruzadas así como los tiempos que debe durar la administración de los diferentes componentes sanguíneos tanto hematíes como plaquetas, y en similares porcentajes los profesionales conocen cuál es el tubo que han de utilizar para realizar dichas pruebas cruzadas. Consideramos que el $100 \%$ de los profesionales debería conocer este hecho para garantizar la seguridad del paciente y prestar un servicio de alta calidad, ahorrando costes derivados por las nuevas extracciones.

En cuanto a la administración conjunta de los concentrados de hematíes con otro tipo de soluciones, tres cuartas partes indican que sólo se puede administrar de forma conjunta con suero fisiológico y una mínima parte indica que además se puede administrar con Voluven®. La bibliografía consultada recomienda estos hechos, en los que indica que se puede administrar de forma conjunta con suero fisiológico y con Voluven®. En el mismo sentido los profesionales indican que el paciente no es necesario que permanezca en ayunas mientras se le realiza la transfusión.

Llama la atención que sólo la mitad de los profesionales indican que los diferentes componentes sanguíneos los administran según llegan del banco de sangre, tal y como se recomienda ${ }^{4}$, y estos esperan a que adquieran temperatura ambiente. Decir que los diferentes componentes sanguíneos han de ser administrados inmediatamente que son recibidos del banco de sangre, puesto que desde el mismo se envían a la temperatura idónea para ser administrados.

Observamos que ante la presencia de fiebre por parte del paciente antes de iniciar transfusión un mínimo porcentaje de los profesionales iniciaría dicha administración, sin embargo, la bibliografía consultada indica que sí se puede iniciar, por el contrario, cuando la fiebre concurre después de iniciar la administración de la transfusión algo más de la mitad pararía la transfusión, aquí la evidencia científica concluye que ha de interrumpirse de forma inmediata. 
En cuanto a las medidas a realizar ante una reacción transfusional, la mayoría de los profesionales tienen claro cuáles son la medidas a realizar, aunque por su gran importancia nos hubiese resultada más gratificante que todos los profesionales conociesen dichas actuaciones.

Teniendo en cuenta todo lo expuesto anteriormente consideramos que la elaboración e implantación de una guía de actuación en cuanto a la administración de hemoderivados se hace imprescindible en nuestro centro.

\section{CONCLUSIONES.}

Por todo lo expuesto anteriormente, consideramos imprescindible desarrollar un protocolo (ANEXO II) para consensuar todos los aspectos en torno a los hemoderivados, que el profesional de enfermería debe llevar a cabo en la práctica diaria de su trabajo para proporcionar unos cuidados de alta calidad y conservar al máximo la seguridad del paciente.

\section{REFEERENCIAS}

1. Torné Pérez $\mathrm{E}$ et al. Fiabilidad del hematocrito postransfusional. Enferm Intensiva 2007;18(4):182-6. [consultado 18/05/2013]. Disponible en: http://www.elsevier.es/es/revistas/enfermeria-intensiva-142/fiabilidad-hematocritopostransfusional-13113137-originales-2007

2. REAL DECRETO 1088/2005, de 16 de septiembre, por el que se establecen los requisitos técnicos y condiciones mínimas de la hemodonación y de los centros y servicios de transfusión. BOE no 225 [consultado 18/05/2013]. Disponible en: http://www.msc.es/profesionales/saludPublica/medicinaTransfusional/legislacion/doc S/RD 1088-2005.pdf

3. REAL DECRETO 478/ 1993, de 2 de abril, por el que se regula los medicamentos derivados de la sangre y plasma humano. BOE № 109 [consultado 18/05/2013]. Disponible en: https://www.boe.es/boe/dias/1993/05/07/pdfs/A1368413687.pdf

4. Ortiz P, et al. Guía sobre la transfusión de componentes sanguíneos. Sociedad Española de Transfusión Sanguínea. Madrid. España. Med Clin (Barc). 2005;125(10):389-96 [consultado 18/05/2013]. Disponible en: http://www.elsevier.es/es/revistas/medicina-clinica-2/guia-transfusion-componentessanguineos-13079172-conferencia-consenso-2005

5. Romero Ruiz A, Gómez Salgado J. Seguridad del paciente en la transfusión sanguínea. Metas de Enferm dic 2008/ene 2009; 11(10): 28-32. [consultado 18/05/2013]. Disponible en: http://www.carloshaya.net/biblioteca/boletinenfermeria5p1/metasseguridaddelpacient e.pdf

6. Plan estratégico de seguridad del paciente. SESCAM. 2009-2012. [consultado 18/05/2013]. Disponible en: http://sescam.jccm.es/web1/ciudadanos/elSescam/Plan Estrategico Seguridad SE SCAM 09-12.pdf

7. ORDEN SCO/322/2007, de 9 de febrero, por la que se establecen los requisitos de trazabilidad y de notificación de reacciones y efectos adversos graves de la 
sangre y de los componentes sanguíneos. [consultado 18/05/2013]. Disponible en: http://www.boe.es/boe/dias/2007/02/17/pdfs/A07010-07016.pdf

8. McClelland DBL et al. Manual de uso óptimo de Componentes sanguíneos. Ministerio De Sanidad, Política Social E Igualdad. Madrid; 2011. [consultado 18/05/2013]. Disponible en: http://www.msps.es/profesionales/saludPublica/medicinaTransfusional/congresos/Jor nadaUsoOptimoComponentesSanguineos/docs/Manual Uso Optimos.pdf

9. Sociedad Española de Transfusión Sanguínea y Terapia Celular. Guía sobre la transfusión de componentes sanguíneos y derivados plasmáticos. Barcelona. $4^{a}$ Edición 2010. [consultado 18/05/2013]. Disponible en: http://www.sets.es/index.php?option=com content\&view=article\&id=446:quiacc\&catid=43: guias\&ltemid $=41$

10. Esteve J. et al. Protocolo general de transfusión de hematíes. Hospital Clinic Barcelona. 2008. [consultado 18/05/2013]. Disponible en: http://anestesiaclinic.net/documents/coagulacio/protocologrltransfhematies.pdf

11. Montoya González M.C. Ezpeleta Irazoz I. Guía de transfusión de componentes sanguíneos en adultos. Servicio Navarro de Salud. [consultado 18/05/2013]. Disponible en: http://www.cfnavarra.es/salud/PUBLICACIONES/Libro\%20electronico\%20de\%20tem as\%20de\%20Urgencia/10.Hematologicas/Terapia\%20transfusional.pdf

12. Comisión de Cuidados del Área de Salud de Badajoz. Transfusión de sangre y hemoderivados. Abril de 2009. [consultado 18/05/2013]. Disponible en: http://www.areasaludbadajoz.com/datos/atencion hospitalaria/Trasfusion\%20sangui nea.\%20Abril\%202009.V.1.1.pdf

13. Hospital General Universitario Gregorio Marañón. Transfusiones de sangre y Hemoderivados. 2009.

14. Encinas Sánchez G. et al. Manual de Protocolos y procedimientos generales de enfermería. Transfusiones y Hemoderivados. Hospital Universitario Reina Sofía. 2010. [consultado 18/05/2013]. Disponible en: http://www.juntadeandalucia.es/servicioandaluzdesalud/hrs3/fileadmin/user upload/a rea enfermeria/enfermeria/procedimientos/procedimientos 2012/rt17 transfusion $\mathrm{h}$ emoderivados.pdf

15. Hernández de León Castro $\mathrm{N}$ et al. Guía clínica de transfusión de componentes sanguíneos. Complejo Hospitalario Universitario de Albacete. [consultado 18/05/2013]. Disponible en: http://www.chospab.es/area medica/banco sangre/GUIA CLINICA DE TRANSFU SION.pdf

16. McCloskey-Dochterman J, Bulechek GM, Cobos Serrano JL et al, editores. Clasificación de Intervenciones de Enfermería (NIC). 4ª Edición. Madrid: Elsevier. 2005.

17. Vázquez Chozas JM, Muñoz González A. El Proceso de Atención de Enfermería. Teoría y Práctica. Madrid: Editorial MAD S.L. 2ª Edición. 2008. 


\section{ANEXO I}

EN TORNO A LOS HEMODERIVADOS

Con el siguiente cuestionario queremos conocer como se realiza la administracción de hemoderivados en muestro centro hospitalario, la finalidad del cuestionario NO es para dar una calificación, el objetivo es hacer una descripción de la situación. Todos los datos recogidos en el cuestionario serán tratados con la míxima confidencialidad garantizando el total anonimato y serán urilizados sólo para el propósito de este estudio. Se ruega la cumplimentación de forma INDIVIDUAL, para rellenarlo indique la respuesta de cómo realizas la técnica, considerando que pueden existir varias respuestas en una misma pregumta. Existen algumas preguntas de contestación obligataria. Muchas gracias por la colaboración.

¿Edad?

¿Sero?

Varón $\quad$ Mujer

¿Experiencia como DUE? (Indicar años) (")

¿Servicio de trabajo habitual? $\left.{ }^{(}\right)$
$\square$ Hospitalización
$\square$ Consultas externas
$\square$ UCI Urgencias/Quirófano
$\square$ Hospital de dia
$\square$ Otro (por favor, especifique)




\section{ANEXO II}

\section{PROCEDIMIENTO: ADMINISTRACIÓN DE PRODUCTOS SANGUÍNEOS. (4030)}

Definición: Administración de sangre y hemoderivados y monitorizar la respuesta del paciente.

La finalidad de la transfusión de hematíes es aumentar la capacidad de transporte de oxígeno a los tejidos gracias a la hemoglobina $(\mathrm{Hb})$.

Los cuidados de enfermería se enmarcan en los establecidos por North American Nursing Diagnosis Association (NANDA), a través de la clasificación de intervenciones de enfermería (NIC), con el objetivo de obtener unos resultados [Clasificación de los resultados de Enfermería (NOC) $]^{16,17}$

1. NANDA : 00146 ANSIEDAD $r / c$ cambio en el estado de salud ( DOMINIO 9 : AFRONTAMIENTO/TOLERANCIA AL ESTRÉS)

- NOC: 1402 Control de la ansiedad

- NIC :

$\checkmark 5820$ Disminución de la ansiedad

2. NANDA : 00093 FATIGA r/c malestar físico ( DOMINIO 4: ACTIVIDAD/REPOSO)

- NOC : 00006 Energía Psicomotora

- NIC :

$\checkmark 0180$ Manejo de la energía

$\checkmark 1803$ Ayuda con los autocuidados : Alimentacion

$\checkmark 1802$ Ayuda con los autocuidados : Vestir/Arreglo personal

$\checkmark 1801$ Ayuda con los autocuidados : Baño/Higiene

$\checkmark 1804$ Ayuda con los autocuidados: eliminación

$\checkmark \quad 1850$ Fomentar el sueño

3. NANDA : 00126 Conocimientos deficientes relacionados con el régimen terapéutico (DOMINIO 5 : PERCEPCION/COGNICIÓN)

- NOC: 1813 Conocimiento : Régimen Terapéutico

- NOC: 3002 Satisfacción del paciente/usuario : Comunicación

- NIC :

$\checkmark \quad 5520$ Facilitar el aprendizaje

$\checkmark 5618$ Enseñanza : Procedimiento/ Tratamiento

4. NANDA : 00206 Riesgo de shock $\mathrm{r} / \mathrm{c}$ el procedimiento terapéutico (DOMINIO 11: SEGURIDAD/PROTECCIÓN)

- NOC : 0802 Signos vitales

- NIC :

$\checkmark 6610$ Identificación de riesgos 
5. NANDA : 00004 Riesgo de infección $r / c$ acceso venoso ( DOMINIO 11: SEGURIDAD/PROTECCIÓN)

- NOC : 1101 Integridad tisular : Piel y membranas mucosas

- NIC :

$$
\begin{array}{ll}
\checkmark & -6540 \text { Control de Infecciones } \\
\checkmark & -6650 \text { Vigilancia } \\
\checkmark & -3590 \text { Vigilancia de la piel }
\end{array}
$$

\section{INDICACIONES:}

Las indicaciones para la administración de concentrados de hematíes son las recogidas en la Tabla I.

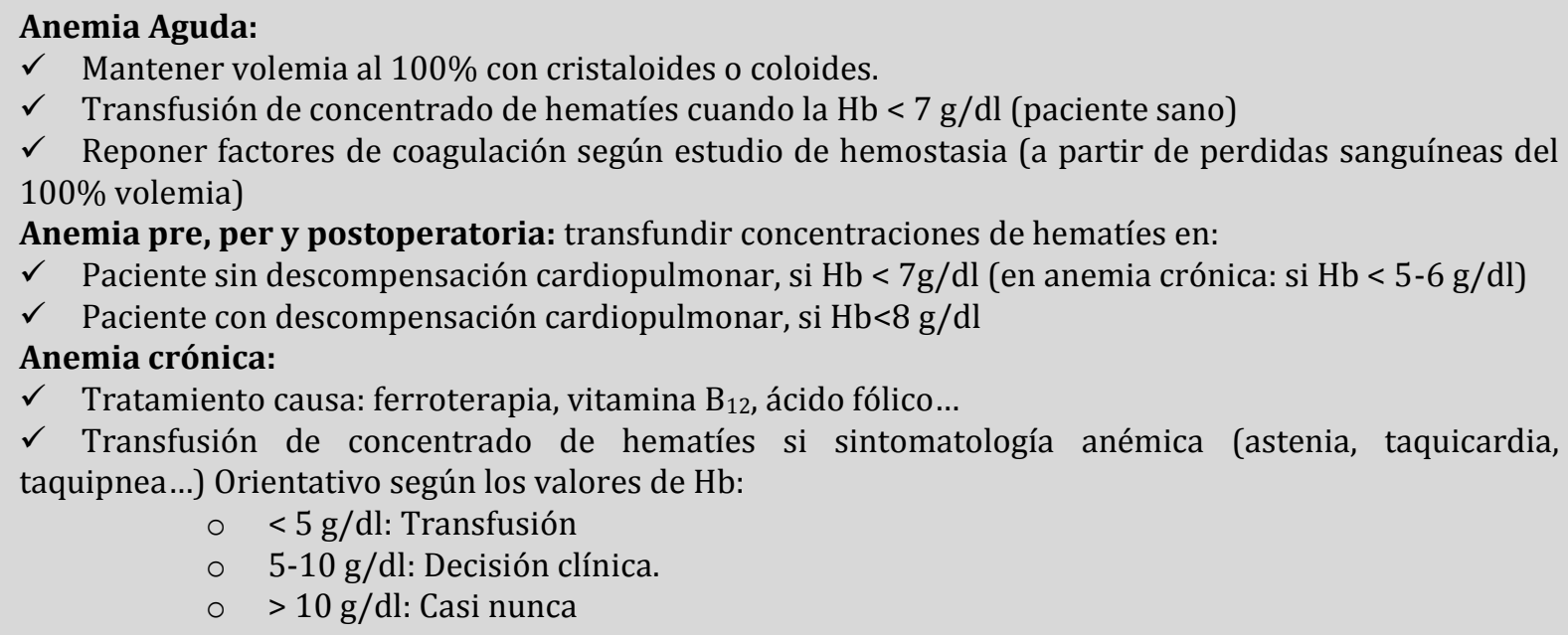

Hb: Hemoglobina

Algunas de las recomendaciones en torno a los hemoderivados son las que a continuación se detallan, algunas de las mismas con marcada evidencia científica $8,9,10,11,12,13,14,15$ :

\section{A. Pretrasfusional.}

\section{CONSENTIMIENTO INFORMADO}

Antes de solicitar la transfusión de componente sanguíneo (CS), siempre que sea posible, el médico que la indica informará al paciente sobre los riesgos y beneficios de la misma así como las posibles alternativas, y recabará su consentimiento informado. El consentimiento informado o la razón por la que no se recabó deberán registrarse en la historia clínica del paciente. (RD 1088/2005 de 16 de Septiembre).

\section{FORMULARIO DE SOLICITUD.}

La solicitud de transfusión debe entenderse como una prescripción médica, estando regulada por la legislación vigente (RD 1088/2005). Por este motivo debe cumplimentarse por personal médico en todos los campos. 
Se realizara únicamente empleando el impreso destinado a tal efecto, debiendo constar, como mínimo, los siguientes datos:

$\checkmark$ Datos de filiación del paciente o pegatina identificativa.

$\checkmark$ Servicio y unidad desde donde se solicita la transfusión.

$\checkmark$ Fecha de la solicitud.

$\checkmark$ Productos a transfundir y cantidad: tipo de CS, especificando si se precisa alguna modificación y cantidad.

$\checkmark$ Grado de urgencia (régimen de transfusión).

$\checkmark$ Motivo que justifica la transfusión.

$\checkmark$ Identificación del médico solicitante.

\section{MUESTRA DE SANGRE}

Junto con la solicitud de transfusión, se entregara una muestra para la realización de las pruebas de compatibilidad. Consistirá en:

Un tubo de sangre total anticoagulada con EDTA de $10 \mathrm{cc}$.

La muestra no debe estar diluida por fluidos endovenosos que esté recibiendo el paciente. Si se obtiene de una vía central o periférica, será necesario desechar los primeros $10 \mathrm{ml}$.

\section{B. Trasfusión.}

\section{TRANSFUSIONES DE CH.}

El resultado de la prueba de compatibilidad tiene una validez de $48 \mathrm{~h}$ desde la extracción de la muestra de sangre del paciente.

\section{TRANSFUSIONES DE CONCENTRA DE PLAQUETAS (CP) Y PLASMA} FRESCO CONGELADO (PFC).

Para trasfundir estos CS, únicamente es necesario conocer el grupo del paciente (tiempo aproximado: 6 minutos). Aunque las pruebas de compatibilidad para estas transfusiones son rápidas, en el caso del plasma hay que descongelarlo previamente, para lo que necesita como mínimo 15 minutos.

\section{SECUENCIA DE LA TRANSFUSION.}

\section{1을 Actuaciones previas.}

$\checkmark$ Comprobar la solicitud de CS y que este correctamente cumplimentada.

$\checkmark$ Confirmar el régimen de transfusión indicado: el/la DUE debe ser consciente de cuál es la situación clínica del paciente así como la urgencia de la transfusión.

$\checkmark$ Cumplimentar la pulsera de seguridad y etiqueta especifica de pruebas cruzadas.

$\checkmark$ Comprobar activamente la identidad del paciente antes de la extracción de la muestra. 


\section{2ㅇtención de la muestra para las pruebas de compatibilidad.}

$\checkmark$ Vía periférica: se canalizará una vía periférica según el protocolo y se realizará una extracción de la muestra.

$\checkmark$ Una vez extraída la muestra se colocará en el tubo la pegatina de la pulsera que se corresponde a la muestra y la etiqueta específica debidamente cumplimentada, procurando que quede visible el contenido del tubo.

$\checkmark$ Se colocará al paciente la pulsera de seguridad.

$\checkmark$ La tira de etiquetas con el código de seguridad sobrante de la pulsera se dejar adherida a la solicitud de transfusión.

\section{infusión:}

3o Antes de iniciar la transfusión y abrir la unidad con el sistema de

$\checkmark$ Es OBLIGATORIO volver a identificar activa e inequívocamente al receptor: solicitando que nos diga su nombre y dos apellidos.

$\checkmark$ La correcta identificación del receptor es MUY IMPORTANTE pues los errores en esta fase son la causa principal de accidentes transfusionales graves.

$\checkmark$ Comprobar la compatibilidad de la transfusión de los CS con otros fluidos que se estén administrando. En caso de vía central con varios accesos, se dejara libre la luz distal, lavándola previamente con solución salina fisiológica, para utilizarla en la administración del CS.

$\checkmark$ Control de constantes: Es importante conocer la tensión arterial, pulso y temperatura antes de administrar cualquier componente sanguíneo. Para evitar desechar CS, este control de constantes debe realizarse antes de que se le aplique el sistema a la unidad.

$\checkmark$ Comprobar el componente sanguíneo:

$>\quad$ Aspecto (que no haya agregados o hemolisis en los $\mathrm{CH}$, en el caso del plasma que esté totalmente descongelado).

$>\quad$ Integridad de la unidad

$>\quad$ Caducidad del producto a transfundir.

$>\quad$ Verificar nuevamente que el componente sanguíneo indicado va a ser administrado al receptor correcto.

\section{4ํAtención al paciente durante la transfusión}

$\checkmark$ Se comenzará la transfusión con un ritmo lento. Cuando se haya comprobado que no provoca ningún efecto adverso se puede aumentar la velocidad (siempre que no haya orden médica en contra).

$\checkmark$ Informar al paciente de la necesidad de comunicar a la enfermera cualquier incidencia observada en el curso de la transfusión.

$\checkmark$ Una vez iniciada la transfusión, controlar al paciente durante los primeros 5-10 minutos para verificar que no presenta ninguna reacción. Posteriormente, continuar observándolo con cierta frecuencia.

$\checkmark$ El/la DUE responsable del paciente tomará las constantes vitales (pulso, temperatura y presión arterial) a los 10 minutos del inicio y al final de esta.

$\checkmark$ También se deberán tomar las constantes siempre que aparezca algún síntoma o se considere medicamente indicado. 


\section{5o Otras consideraciones:}

$\checkmark$ El paciente puede comer y beber líquidos durante la transfusión (salvo indicación en facultativa en contra).

$\checkmark$ La presencia de fiebre no contraindica la transfusión, sin bien es recomendable esperar a que se normalice la temperatura, ya que la fiebre durante la transfusión puede alertar de una posible reacción transfusional.

$\checkmark$ En los pacientes adultos normovolémicos puede valorarse realizando un control de las concentraciones de $\mathrm{Hb} /$ hematocrito a partir de los $15 \mathrm{~min}$ de finalizar la transfusión4

$\checkmark$ Hematocrito postransfusional no sufre variaciones significativas con el transcurso del tiempo. O lo que es lo mismo, que la determinación inmediata del hematocrito es tan fiable como la que realicemos a la hora o posterior.

\section{INMEDIATA}

ACTUACION DE ENFERMERIA ANTE UNA REACCION TRANSFUSIONAL

1. Detener la transfusión, para limitar la cantidad de componente infundido.

2. Mantener la vía con solución salina $0,9 \%$.

3. Avisar al médico responsable del paciente.

4. Verificar todos los registros, las etiquetas e identificaciones del producto transfundido y del paciente para determinar que este ha recibido el componente previsto. diuresis.

5. Control de temperatura, tensión arterial, frecuencia cardiaca y respiratoria y

6. Enviar al Servicio de Transfusión, lo antes posible, la bolsa causante de la reacción sin quitar el sistema de transfusión, junto con el impreso de Notificación de reacción transfusional debidamente cumplimentado por el médico responsable del paciente y una muestra de sangre en tubo de 10cc con EDTA (malva)6.

\section{INFUSION DE LOS COMPONENTES}

Si fuera necesario transfundir varios CS, el orden de los mismos será:

1. Plasma fresco

2. Plaquetas

3. Concentrado de Hematíes.

Se evitará la infusión de dichos CS de forma simultánea, y la modificación de dicho orden versará bajo prescripción facultativa.

\section{VELOCIDAD DE INFUSION}

Se ajustará a las necesidades del paciente. La velocidad de infusión podrá ser menor en niños, ancianos, pacientes con insuficiencia cardiaca o insuficiencia renal, o cuando así lo indique el médico responsable del paciente.

Los primeros minutos de cualquier transfusión (exceptuando en las situaciones de sangrado severo) debe realizarse a velocidad lenta ( $2 \mathrm{ml} / \mathrm{min}$ o $10 \mathrm{gotas} / \mathrm{min})$. Solo 
cuando se haya comprobado que, tras 5-10 minutos, no se ha producido reacción se puede pasar a los flujos correspondientes de cada componente (Tabla 2):

\begin{tabular}{|c|c|c|c|c|}
\hline \multicolumn{5}{|c|}{ Tabla 2} \\
\hline Producto & Volumen & Conservación & $\begin{array}{c}\text { Duración } \\
\text { de la } \\
\text { transfusión }\end{array}$ & $\begin{array}{l}\text { Ritmo de } \\
\text { infusión }\end{array}$ \\
\hline HEMATIES & $\begin{array}{c}300-400 \\
\mathrm{ml}\end{array}$ & 2-6C: 42 días & $60-120 \mathrm{~min}$ & $\begin{array}{l}30-60 \\
\text { gotas/min }\end{array}$ \\
\hline PLASMA & $\begin{array}{c}200-300 \\
\mathrm{ml}\end{array}$ & $\begin{array}{l}\text { Congelado }\left(-25^{\circ} \mathrm{C}\right): 24 \\
\text { meses } \\
\text { Descongelado }\left(2-6^{\circ} \mathrm{C}\right): \\
24 \text { horas }\end{array}$ & 20-30 min & $\begin{array}{l}125-175 \\
\text { gotas/min }\end{array}$ \\
\hline PLAQUETAS & $\begin{array}{c}200-300 \\
M L\end{array}$ & $\begin{array}{l}22^{\circ} \mathrm{C} \text { en agitación } \\
\text { continua: } 5 \text { días }\end{array}$ & 20-30 min & $\begin{array}{l}\text { 125-225 gotas/ } \\
\text { nin }\end{array}$ \\
\hline
\end{tabular}

En condiciones normales, un $\mathrm{CH}$ en un adulto debe pasar en 1-2 horas, y nunca en más de 4 horas. Si existe sobrecarga hídrica se recomienda un ritmo de 1 $\mathrm{ml} / \mathrm{kg} /$ hora. Si las circunstancias del paciente obligan a disminuir tanto la velocidad de infusión que el $\mathrm{CH}$ haya de permanecer más de 4 horas a temperatura ambiente, se solicitara al Servicio de Transfusión que fraccione la unidad de $\mathrm{CH}$ en varias.

Las transfusiones de plaquetas se realizarán tan rápidamente como sea tolerada por el receptor, por lo general entre 20 y 30 minutos. En caso de riesgo de sobrecarga hídrica, se enlentecerá el ritmo de infusión.

Recibido: 16 de agosto de 2013; Aceptado: 4 de noviembre de 2013 